\title{
COMMENTARY
}

\section{The role of lactate clearance in the resuscitation bundle}

\author{
Anthony M Napoli* and Todd A Seigel \\ See related research by Nguyen et al., http://ccforum.com/content/15/5/R229
}

\begin{abstract}
The sepsis resuscitation bundle is the result of an effort on behalf of the Surviving Sepsis Campaign and the Institute for Healthcare Improvement to translate individual guideline recommendations into standardized, achievable goals for physicians caring for the critically ill patient. Implementation of this bundle is associated with decreased mortality. Many of the bundle items reflect components of therapy shown to improve mortality in the seminal early goaldirected therapy trial for severe sepsis and septic shock, including an initial lactate measurement. Elevations in serum lactate are associated with increased mortality, and may result from either increased lactate production or impaired lactate clearance. Lactate clearance may be an important addition to the monitoring and management bundles of patients with severe sepsis and septic shock, However, specific mechanisms of lactate clearance, the relation of lactate clearance to traditional hemodynamic parameters, and the importance of lactate clearance as a therapeutic target or monitoring tool remain unclear.
\end{abstract}

In the present issue of Critical Care, Nguyen and colleagues investigate the utility of adding lactate clearance into the Surviving Sepsis Campaign (SSC) resuscitation bundle in order to assess the relative effectiveness of this modified bundle in predicting mortality in patients with severe sepsis and septic shock [1]. Implementation of the SSC bundle has been shown to be associated with a decrease in mortality [2-4].

Lactate clearance, generally represented as the percentage reduction in lactate over a resuscitation interval, has also been associated with decreased mortality in patients

*Correspondence: anapoli@lifespan.org

Department of Emergency Medicine, Warren Alpert School of Medicine at Brown University, 593 Eddy Street, Davol 142, Providence, RI 02903, USA with severe sepsis and septic shock [5,6] as well as with multiple organ dysfunction and systemic immunologic activation and inflammation [7]. While early resuscitation of patients with severe sepsis and septic shock focuses on optimizing hemodynamics and oxygen delivery [8], lactate may be an indicator of impaired microcirculatory perfusion and often is the only indicator of severe sepsis [9]. However, lactate clearance is closely associated with capillary perfusion independent of hemodynamic variables [10]. A recent randomized controlled trial of lactate monitoring and therapy targeted toward lactate clearance improved outcome in ICU patients with hyperlactatemia [11].

Since both implementation of the SSC bundle and lactate clearance have independently been associated with a decrease in mortality [2-6,11], incorporating lactate clearance may represent an opportunity to simultaneously combine the outcome benefit of monitoring the optimization of both global hemodynamics and microvascular perfusion.

This multinational cooperative represents the first initiative to study the addition of lactate clearance into the SSC bundle to create a modified bundle and compare the mortality with successful completion of each of the two bundles. The standard SSC bundle includes seven parameters: initial lactate measurement, blood cultures prior to antibiotics, antibiotics administered by 3 hours, initiation of fluid bolus, and central venous pressure $>8 \mathrm{mmHg}$, mean arterial pressure $>65 \mathrm{mmHg}$ and central venous oxygen saturation $>70 \%$ by 6 hours. Lactate clearance, in this study, was defined as any decrease in lactate within 12 hours or an initial lactate $<2.0 \mathrm{mmol} / \mathrm{l}$ [1]. As expected, completion of the SSC resuscitation bundle was associated with decreased mortality. In comparison with the standard SSC bundle, however, completion of the modified resuscitation bundle was associated with a nearly twofold decrease in the relative risk reduction for mortality. Subsequent multivariate analysis demonstrated an independent association of lactate clearance, administration of a fluid bolus, and mean arterial pressure $>65 \mathrm{mmHg}$ by 6 hours with decreased mortality (odds ratio of $0.32,0.47$, and 0.20 , respectively). 
Nguyen and colleagues' study suggests a benefit of adding lactate clearance to the standard SSC resuscitation bundle in patients with severe sepsis [1]. However, the relationship of lactate clearance to global hemodynamics and the degree to which hemodynamic optimization impacts lactate clearance remain uncertain. In this investigation, baseline markers of global perfusion (central venous pressure, mean arterial pressure, and central venous oxygen saturation) were near normal prior to initiation of either resuscitation bundle, suggesting a better resuscitated, less supply-dependent population than the original early goal-directed therapy study. In addition, baseline hemodynamics, vasopressor use, and the amount of fluid resuscitation were similar for both patients who cleared lactate and those who did not.

These results reflect previous studies that demonstrate discordance between lactate clearance and traditional hemodynamic markers [12]. Despite relatively similar hemodynamics and clinical interventions, patients who did not adequately clear lactate had higher initial lactate levels and baseline Acute Physiology and Chronic Health Evaluation scores than those who did. This suggests a mechanism of lactate clearance unrelated to adequacy of global perfusion, and it remains unclear whether the ability to clear lactate is related to achieving broader hemodynamic goals. In addition, this study includes patients with an initial lactate $<2 \mathrm{mmol} / \mathrm{l}$ in the same cohort as patients initially with hyperlactatemia who eventually clear their lactate, making it difficult to evaluate the differences between those groups and the role of lactate clearance in each population. It is known that alactemic patients with hypotension represent up to one-third of patients diagnosed with septic shock but these patients have much lower mortality [13]. Despite this, poor lactate clearance appears to have prognostic value in normolactatemic and hyperlactatemic patients $[14,15]$ and may stem from a defect in lactate utilization rather than lactate overproduction. Regardless, the present study contextualizes the importance of lactate clearance by suggesting persistent hyperlactatemia may be independent of other hemodynamic factors and portends a worse prognosis.

Ultimately, the degree to which lactate clearance is dependent on adherence to standardized bundles is uncertain. Lactate clearance may be specific to the patient, to the severity of the acute illness, or to the stage of illness. The etiology of persistent hyperlactatemia may be multifactorial, accompanying a hypovolemic stage, a compensatory or vasodilatory stage, myocardial suppression, or impaired tissue oxygen utilization after hemodynamic optimization. It is in this latter stage where lactate clearance may be particularly helpful in identifying those patients at continued increased risk of mortality after aggressive resuscitation measures are met.
Moving forward, lactate clearance may have particular utility in noninvasive resuscitation protocols where hemodynamics do not appear compromised, but where impaired clearance may direct more invasive, aggressive measures. Research into patient-specific or diseasespecific factors that affect lactate clearance as well as targeted interventions toward improving lactate utilization, independent of hemodynamic optimization, may be the next step in improving the care of these patients.

\section{Abbreviations}

SSC, Surviving Sepsis Campaign.

\section{Competing interests}

The authors declare that they have no competing interests.

\section{Published: 24 October 2011}

\section{References}

1. Nguyen HB, Kuan WS, Batech M, Shrikhande P, Mahadevan M, Li C, Ray S, Dengel A: Outcome effectiveness of the severe sepsis resuscitation bundle with addition of lactate clearance as a bundle item: a multi-national evaluation. Crit Care 2011, 15:R229.

2. Levy MM, Dellinger RP, Townsend SR, Linde-Zwirble WT, Marshall JC, Bion J, Schorr C, Artigas A, Ramsay G, Beale R, Parker MM, Gerlach H, Reinhart K, Silva E, Harvey M, Regan S, Angus DC: The Surviving Sepsis Campaign: results of an international guideline-based performance improvement program targeting severe sepsis. Crit Care Med 2010, 38:367-374.

3. Coba V, Whitmill M, Mooney R, Horst HM, Brandt MM, Digiovine B, Mlynarek M, McLellan B, Boleski G, Yang J, Conway W, Jordon J: Resuscitation bundle compliance in severe sepsis and septic shock: improves survival, is better late than never. J Intensive Care Med 2011, in press. [Epub ahead of print.]

4. Nguyen HB, Corbett SW, Steele R, Banta J, Clark RT, Hayes SR, Edwards J, Cho TW, Wittlake WA: Implementation of a bundle of quality indicators for the early management of severe sepsis and septic shock is associated with decreased mortality. Crit Care Med 2007, 35:1105-1112.

5. Bakker J, Gris P, Coffernils M, Kahn RJ, Vincent JL: Serial blood lactate levels can predict the development of multiple organ failure following septic shock. Am J Surg 1996, 171:221-226.

6. Nguyen HB, Rivers EP, Knoblich BP, Jacobsen G, Muzzin A, Ressler JA, Tomlanovich MC: Early lactate clearance is associated with improved outcome in severe sepsis and septic shock. Crit Care Med 2004, 32:1637-1642.

7. Nguyen HB, Loomba M, Yang JJ, Jacobsen G, Shah K, Otero RM, Suarez A, Parekh H, Jaehne A, Rivers EP: Early lactate clearance is associated with biomarkers of inflammation, coagulation, apoptosis, organ dysfunction and mortality in severe sepsis and septic shock. J Inflamm (Lond) 2010, 7:6.

8. Rivers E, Nguyen B, Havstad S, Ressler J, Muzzin A, Knoblich B, Peterson E, Tomlanovich M: Early goal-directed therapy in the treatment of severe sepsis and septic shock. N Engl J Med 2001, 345:1368-1377.

9. Puskarich MA, Trzeciak S, Shapiro NI, Heffner AC, Kline JA, Jones AE: Outcomes of patients undergoing early sepsis resuscitation for cryptic shock compared with overt shock. Resuscitation 2011, 82:1289-1293.

10. De Backer D, Creteur J, Dubois MJ, Sakr Y, Koch M, Verdant C, Vincent JL: The effects of dobutamine on microcirculatory alterations in patients with septic shock are independent of its systemic effects. Crit Care Med 2006, 34:403-408.

11. Jansen TC, van Bommel J, Schoonderbeek FJ, Sleeswijk Visser SJ, van der Klooster JM, Lima AP, Willemsen SP, Bakker J: Early lactate-guided therapy in intensive care unit patients: a multicenter, open-label, randomized controlled trial. Am J Respir Crit Care Med 2010, 182:752-761.

12. Arnold RC, Shapiro NI, Jones AE, Schorr C, Pope J, Casner E, Parrillo JE, Dellinger RP, Trzeciak S: Multicenter study of early lactate clearance as a determinant of survival in patients with presumed sepsis. Shock 2009, 32:35-39.

13. Hernandez G, Castro R, Romero C, de la Hoz C, Angulo D, Aranguiz I, Larrondo J, Bujes A, Bruhn A: Persistent sepsis-induced hypotension without hyperlactatemia: is it really septic shock? J Crit Care 2011, 26:435 e9-e14. 
14. Levraut J, Ichai C, Petit I, Ciebiera JP, Perus O, Grimaud D: Low exogenous lactate clearance as an early predictor of mortality in normolactatemic critically ill septic patients. Crit Care Med 2003, 31:705-710.

15. Levraut J, Ciebiera JP, Chave S, Rabary O, Jambou P, Carles M, Grimaud D: Mild hyperlactatemia in stable septic patients is due to impaired lactate clearance rather than overproduction. Am J Respir Crit Care Med 1998, 157(4 Pt 1):1021-1026. doi:10.1186/cc10478

Cite this article as: Napoli A, Seigel TA: The role of lactate clearance in the resuscitation bundle. Critical Care 2011, 15:199. 